\title{
ERUPCIÓN PASIVA ALTERADA: REPORTE DE CASO.
}

\section{ALTERED PASSIVE ERUPTION: A CASE REPORT.}

\author{
Pinos-Ochoa Xavier ${ }^{1 *}$, Ortiz-Garay Elida Magaly ${ }^{2}$ \\ ${ }^{1}$ Odontólogo Epecialista en Periodoncista de consulta particular Cuenca. Ecuador \\ 2 Odontóloga Especialista en Endodoncista de consulta particular Cuenca. Ecuador \\ *xapo7@ hotmail.com
}

\begin{abstract}
Resumen
La erupción pasiva alterada produce un exceso gingival el cual ocasiona pseudobolsas y compromete la estética del paciente. El tratamiento periodontal debe enfocarse en restablecer la relación normal entre el margen gingival, la cresta ósea alveolar y la unión amelocementaria (CAL). El objetivo de este artículo es presentar el manejo clínico (diagnóstico y plan de tratamiento) de la erupción pasiva alterada.
\end{abstract}

Palabras clave: erupción pasiva alterada, sonrisa gingival, tratamiento periodontal.

\begin{abstract}
The altered passive eruption produces a gingival excess which causes pseudo bags and compromises the aesthetics of the patient. The periodontal treatment should focus on restoring the normal relationship between the gingival margin, the alveolar bone crest and the cementoenamel junction $(C A L)$. The objective of this article is to present the clinical management (diagnosis and treatment plan) of the altered passive eruption.
\end{abstract}

Key words: altered passive eruption, excessive display of gingiva, periodontal treatment.

\section{INTRODUCCIÓN}

La erupción pasiva alterada se define cuando los márgenes gingivales de los dientes permanentes no retroceden a nivel del límite amelocementario (CEJ) después de su erupción completa. ${ }^{1-5}$

Clínicamente es una condición de desarrollo que se caracteriza por que el margen gingival (y a veces la cresta ósea) está ubicado a un nivel más coronal. Esta condición, cuya prevalencia es del $12 \%,{ }^{6}$ puede asociarse clínicamente con la formación de pseudobolsas o con compromiso estético. ${ }^{7,8}$

La erupción pasiva alterada se clasifica en dos tipos y dos subgrupos: Tipo 1, cuando el margen gingival se localiza incisal u oclusal con respecto al CEJ y presenta una banda ancha de encía insertada; Tipo2, presenta un ancho normal de la encía insertada $(3,0-4,2 \mathrm{~mm}$ en el maxilar y 2,5-2,6 $\mathrm{mm}$ en la mandíbula) ${ }^{9,10}$ y la unión mucogingival está a nivel del CEJ $;^{2}$ Subgrupo A, la cresta alveolar está a una distancia normal (1-2 mm apical) del CEJ; Subgrupo B, la cresta alveolar se encuentra a nivel del CEJ. ${ }^{2,9}$ El tratamiento de la erupción pasiva alterada es quirúrgico (Gingivectomía/ Colgajo Reposicionado Apical-Osteotomía/ Osteoplastia) y el manejo clínico dependerá de su diagnóstico. ${ }^{11,12}$ A con-

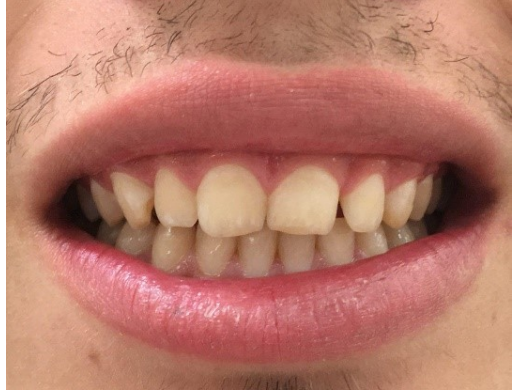

Fig. 1. Sonrisa Normal

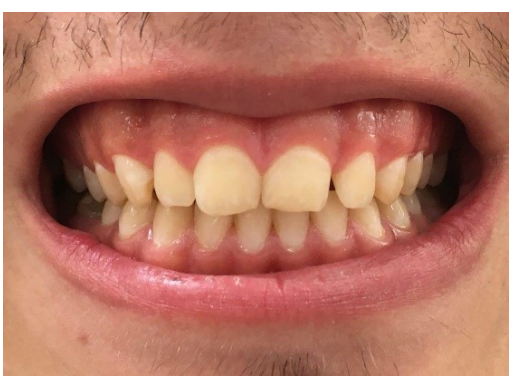

Fig. 2. Sonrisa Forzada 
tinuación, se reporta un caso clínico de erupción pasiva alterada donde se detalla su abordaje.

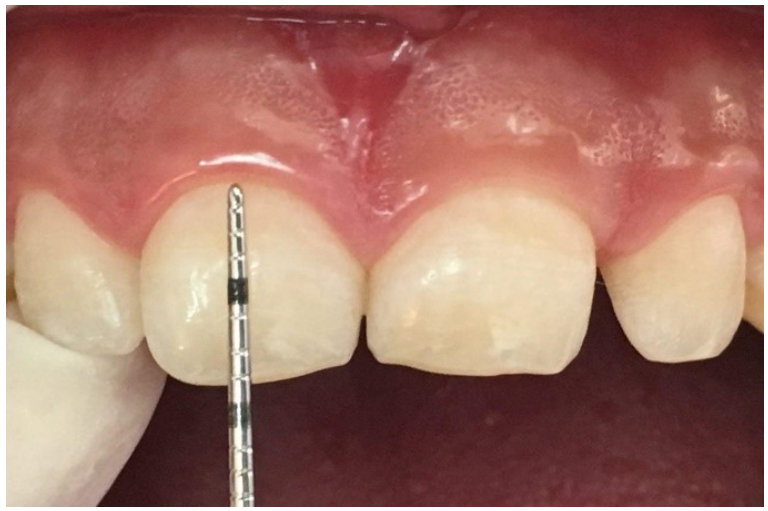

Fig. 3. Corona clínica corta $8 \mathrm{~mm}$

\section{REPORTE DE CASO CLÍNICO}

Paciente de sexo masculino de 21 años de edad que presenta exceso gingival a nivel del sector anterosuperior en sonrisa normal $(3,0 \mathrm{~mm})$ y forzada $(6,0 \mathrm{~mm})$ (Figura 1), (Figura2), coronas clínicas cortas (Figura4), y banda ancha de encía insertada.

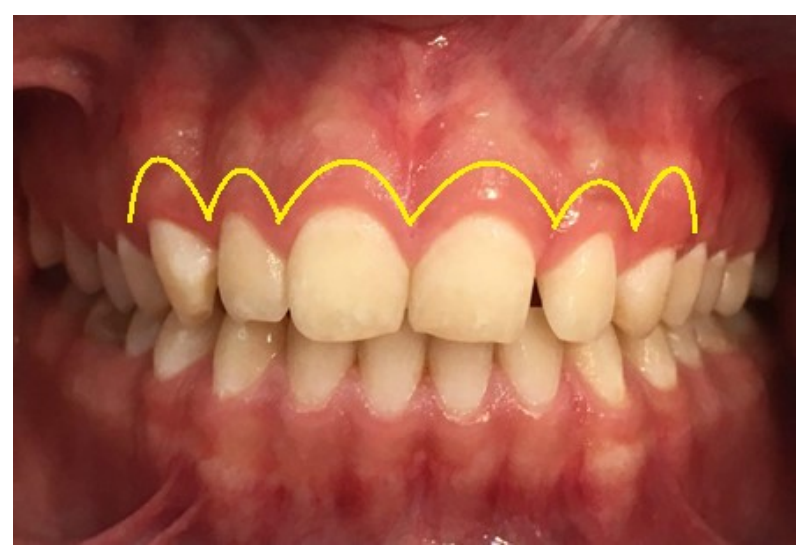

Fig. 4. Planificación

Al sondaje periodontal no se ubica el CEJ, y las radiografías periapicales con paralelismo demuestran que a nivel interdental la cresta ósea se ubica a nivel del CEJ, determinando el diagnóstico de sonrisa gingival por erupción pasiva alterada tipo $1 \mathrm{~B} .{ }^{4,9}$

Planificación: Se mide la corona clínica $(8 \mathrm{~mm})$ y la corona anatómica en base a las radiografías periapicales $(11 \mathrm{~mm})$, existiendo una diferencia de $3 \mathrm{~mm}$; se planifican realizar incisiones festoneadas de $2,5 \mathrm{~mm}$ apical al margen gingival, de canino a canino (Figura 3$).{ }^{13}$

Pre-operatorio: Desinfección intraoral con clorhexidina al $0,12 \%$ por un minuto y desinfección extraoral con Yodopovidona al $10 \% .^{14}$

Operatorio: Se realizan incisiones festoneadas $2,5 \mathrm{~mm}$ apical al margen gingival de canino a canino (Figura 5); se retira el collarín gingival (Figura 5), se realiza el levantamiento de un colgajo mucoperióstico (Figura 5). Se practica una osteotomía de 1,5 a $2 \mathrm{~mm}$ de distancia entre la cresta ósea y el CEJ (Figura 5), seguida de una osteoplastía para mejorar el contorno óseo. Finalmente se efectúa el reposicionamiento apical del colgajo, seguido de sutura interrumpida simple en cada papila (Figura 5). ${ }^{15-17}$

Post-operatorio: Se receta antinflamatorio: etoricoxib de $120 \mathrm{mg}$; 1 cada 24 horas por 5 días. Se recomienda dieta blanda, crioterapia, y enjuague bucal con clorhexidina $0,12 \%$ $15 \mathrm{ml}$ por 1 minuto cada 12 horas por 2 semanas. ${ }^{13}$

Se retiran los puntos a los 10 días, y se realiza control a los 3 , 6 y 9 meses (Figura 6).

Se observa la ganancia de corona clínica de $2 \mathrm{~mm}$ y márgenes gingivales que se mantienen en su sitio a los 9 meses (Figura 6).

\section{DISCUSIÓN}

La mayoría de los estudios que investigan la erupción pasiva alterada son de tipo preliminar, y se caracterizan por incluir grupos pequeños de pacientes. Hasta donde sabemos, no se han publicado artículos sobre investigaciones que evalúen cambios en la longitud de la corona clínica a largo plazo.

Las dificultades para distinguir la erupción pasiva alterada perteneciente al subgrupo A o el subgrupo B, junto con la presencia de diferentes subgrupos de erupción pasiva alterada en la misma área quirúrgica, complican la planificación del tratamiento quirúrgico. Frente a alteraciones menores de la armonia del margen de tejido blando, la gingivectomía es el tratamiento de elección. Sin embargo, la mayoría de los casos de erupción pasiva alterada requieren de osteotomía y/u osteoplastia. La principal discrepancia entre los autores se relaciona con la distancia correcta entre la cresta ósea y el CEJ (que va de 1 a $3 \mathrm{~mm}$ ) en pacientes con erupción pasiva subgrupo B. ${ }^{13,15}$

Esta distancia es crítica para determinar la cantidad de osteotomía que va a realizarse durante la cirugía. Esto se debe a la alta variabilidad en el ancho de la unión dentogingival humana. ${ }^{18}$

Sin embargo, debido a que esta distancia debe reflejar el área ocupada solo por la inserción conectiva, no debe ser mayor de 1-1,5 mm. ${ }^{19}$ Esta área no debe ser instrumentada (raspado y alisado radicular) durante la cirugía, ya que se podría producir pérdida de inserción conectiva y hueso. ${ }^{2}$ 

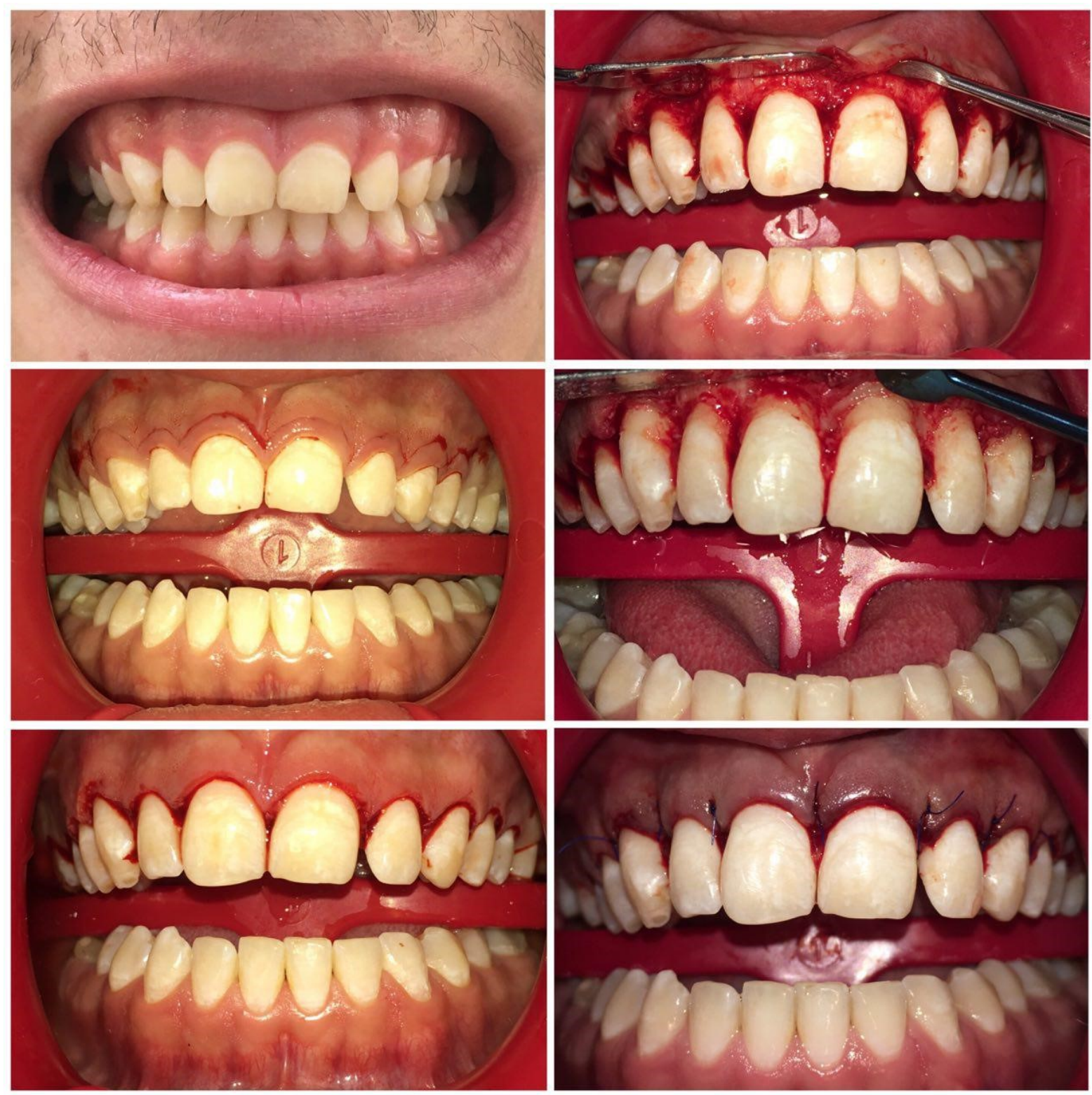

Fig. 5. Manejo Quirúrgico 

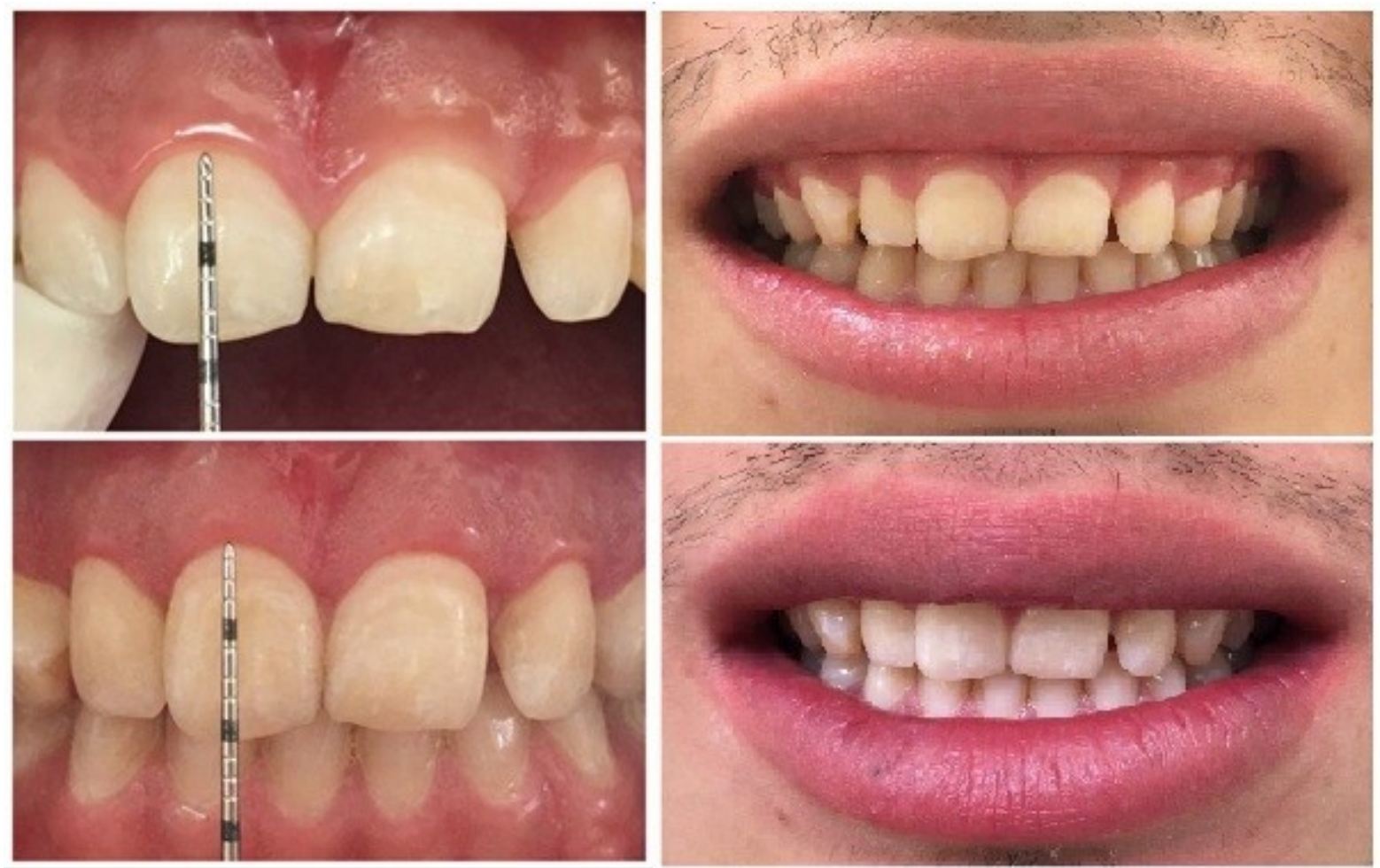

Fig. 6. Antes y después de 9 meses 


\section{CONCLUSIÓN}

El objetivo del tratamiento de la erupción pasiva alterada es mejorar la apariencia y sonrisa del paciente, elevando su autoestima. Este procedimiento se debe realizar sobre la base de un diagnóstico adecuado, y con una buena planificación del tratamiento quirúrgico para que el mismo sea exitoso a largo plazo.

\section{Referencias Bibliográficas}

1 Silberberg N, Goldstein M, Smidt A. Excessive gingival display-Etiology, diagnosis, and treatment modalities. Quintessence International. 2009;40(10):809-818.

2 Mele M, Felice P, Sharma P, Mazzotti C, Bellone P, Zucchelli G. Esthetic treatment of altered passive eruption. Periodontology 2000. 2018;77(1):65-83.

3 Lee EA. Aesthetic crown lengthening: classification, biologic rationale, and treatment planning considerations. Pract Proced Aesthet Dent. 2004;16(10):769-778.

4 Rossi R, Benedetti R, Santos R. Treatment of altered passive eruption: periodontal plastic surgery of the dentogingival junction. European Journal of Esthetic Dentistry. 2008;3(3).

5 Ahmad I. Altered passive eruption (APE) and active secondary eruption (ASE): differential diagnosis and management. The international journal of esthetic dentistry. 2017;12(3):352-376.

6 Volchansky A, Cleaton-Jones P. Delayed passive eruptionA predisposing factor to Vincent's Infection?. Journal of the D.A.S.A. 1974; 29( 5): 291- 294.

7 Caton JG, Armitage G, Berglundh T, Chapple IL, Jepsen $\mathrm{S}$, Kornman KS, et al. A new classification scheme for periodontal and peri-implant diseases and conditions- Introduction and key changes from the 1999 classification. Journal of periodontology. 2018;89:S1-S8.

8 Jepsen S, Caton JG, et al. Periodontalmanifestations of systemic diseases and developmental andacquired conditions: Consensus report of workgroup 3 of the 2017 World Workshop on the Classification of Periodontaland Peri-Implant Diseases and Conditions. J Clin Periodontol. 2018;45(20):S219-S229.

9 Coslet J, Vanarsdall R, Weisgold A. Diagnosis and classification of delayed passive eruption of the dentogingival junction in the adult. The Alpha Omegan. 1977;70(3):24 28.

10 Bowers GM. A study of the width of attached gingiva. The Journal of Periodontology. 1963;34(3):201-209.

11 Alpiste-Illueca F. Altered passive eruption (APE): a littleknown clinical situation. Med Oral Patol Oral Cir Bucal. 2011;16(1):100-104.

12 Marzadori M, Stefanini M, Sangiorgi M, Mounssif I, Monaco $\mathrm{C}$, Zucchelli G. Crown lengthening and restorative procedures in the esthetic zone. Periodontology 2000.

13 Cairo F, Graziani F, Franchi L, Defraia E, Pini Prato GP. Periodontal plastic surgery to improve aesthetics in patients with altered passive eruption/gummy smile: a case series study. International journal of dentistry. 2012; 1-6.

14 Löe H, Anerud A, Boysen H, Morrison E. Natural history of periodontal disease in man: rapid, moderate and no loss of attachment in Sri Lankan laborers 14 to 46 years of age. Journal of clinical periodontology. 1986;13(5):431-440.

15 Silva CO, Soumaille JM, Marson FC, Progiante PS, Tatakis DN. Aesthetic crown lengthening: periodontal and patient-centred outcomes. Journal of clinical periodontology. 2015;42(12):1126-1134.

16 Pulgaonkar R, ChitRa P. Altered Passive Eruption Complicating Optimal Orthodontic Bracket Placement: A Case Report and Review of Literature. Journal of clinical and diagnostic research: JCDR. 2015;9(11): ZD01-ZCD03.

17 Fletcher P. Biologic rationale of esthetic crown lengthening using innovative proportion gauges. International Journal of Periodontics and Restorative Dentistry. 2011;31(5):523.

18 Schmidt JC, Sahrmann P, Weiger R, Schmidlin PR, Walter C. Biologic width dimensions-a systematic review. Journal of clinical periodontology. 2013;40(5):493-504.

19 Gargiulo AW, Wentz FM, Orban B. Mitotic activity of human oral epithelium exposed to 30 per cent hydrogen peroxide. Oral Surgery, Oral Medicine, Oral Pathology. 1961;14(4):474-492.
Recibido: 13 de febrero de 2019.

Aceptado: 7 de Marzo de 2019. 
